\title{
Development of a growth model for aluminum-doped zinc oxide nanocrystal synthesis via the benzylamine route
}

\author{
Julian Ungerer $(\mathbb{D} \cdot$ Ann-Kathrin Thurm • \\ Manuel Meier • Mira Klinge • Georg Garnweitner • \\ Hermann Nirschl
}

Received: 1 March 2019 / Accepted: 2 May 2019

(C) Springer Nature B.V. 2019 kinetics data, further transmission electron microscopy (TEM) and SAXS analyses are performed to investigate changes in terms of shape, size, and fractal properties leading into the development of a generalized growth model for AZO nanocrystals during synthesis.

Keywords AZO nanocrystals - The benzylamine route . Process kinetics · Particle morphology · Growth model of AZO $\cdot$ SAXS $\cdot$ Synthesis

\section{Introduction}

The ever-increasing demand for resources based on expensive rare earth metals requires the availability of cheap alternatives having similar requirements in the field of application. A research focus is therefore the n-type semiconductor aluminum-doped zinc oxide (AZO), which will be seen in the future to replace the previously versatilely used toxic indium-based oxides in diverse scopes, e.g., lightemitting diodes, printing electronics, and transparent conducting oxide (TCO) thin-films in solar modules, due to similar properties, such as low electrical resistance, high degree of transparency in the visible range, and good mechanical stability. On the one hand, particular attention is paid to the field of application in terms of printable electronics, which represents an important research focus due to the favorable availability of tailor-made electronic components (Ismail et al. 2001; Patil et al. 2011; Stubhan et al. 2011). On the other hand, in terms 
of the growing demand for renewable energy resources, another research focus is set on transparent thin-film solar cells based on TCOs (Ellinger and Nelson 2014; Jiang et al. 2003; Luo et al. 2012; Minami 2005; Strachowski et al. 2007). These complex applications requires a comprehensive understanding of the manufacturing process to ensure the availability of high-quality AZO nanocrystals with defined sizes, shapes, and doping levels of aluminum to adjust optical, mechanical, and electrical application properties (Luo et al. 2012; Strachowski et al. 2007). In contrast to gas-phase and solid processes (Yoon et al. 2002) or coprecipitation (Nie et al. 2008), solvent-based liquid-phase syntheses result in better process control at moderate reaction rates (Cushing et al. 2004; Livage et al. 1988). Thus, this synthesis route provides access to measurement technologies in terms of time-resolved analysis of particle formation and growth processes, as we show in this work. For synthesizing highly crystalline metal oxide nanostructures with narrow particle size distributions at moderate temperatures, the non-aqueous sol-gel synthesis is used, wherein an aromatic solvent performs significant functions during the growth process, e.g., a defined influencing of the particle morphology or the control of growth kinetics (Garnweitner et al. 2008; Niederberger 2007; Pinna and Niederberger 2008; Zellmer et al. 2015). In our work, a new concept for time-resolved analysis techniques is established, which provides comprehensive insights into particle formation processes leading to a general growth model of AZO nanocrystals during synthesis. The major challenge is to gain timeresolved access to the synthesis process via the benzylamine route, which we used in this work, by means of measurement technology. The work by Zellmer et al. (2015) has shown time-resolved studies of crystal growth of AZO nanocrystals via the benzylamine route preferably at high temperatures $\left(\sim 200^{\circ} \mathrm{C}\right)$, high precursor concentrations $\left(\sim 50 \mathrm{~g} \mathrm{~L}^{-1}\right)$, and relatively short reaction times $(\sim 30 \mathrm{~min})$. In order to be able to resolve the crystal growth more precisely, longer reaction rates at lower process temperatures are desirable. However, in process control at low process temperatures $\left(\ll 200^{\circ} \mathrm{C}\right)$, physical limits are set, because of strongly decreasing solubility of precursors in the solvent benzylamine and increasing viscosity of the particle dispersion. Furthermore, due to increasing solvent corrosivity with temperature and the strong aggregation tendency of AZO nanocrystals, an access of a measurement technique to the synthesis process is only possible to a very limited extent with conventional measurement methods, such as dynamic lightscattering or transmission electron microscopy (TEM). Taking into account these limitations in nanoparticle analysis mentioned here, a new concept is developed in this work, firstly, to ensure the access of analysis methods to the synthesis at lower process temperature range $T_{\mathrm{R}}<120^{\circ} \mathrm{C}$ by selection of suitable measurement technology and, secondly, to perform time-resolved investigations of ongoing particle growth and consumption processes despite aggregate formation. Particularly suitable for this purpose is the non-invasive small-angle X-ray scattering (SAXS) measurement technique, which is primarily used in this work. A lab-scale SAXS camera is used, on the one hand, performing quantitative phase analysis (QPA) to enable a time-resolved quantitative recording of crystalline materials in liquid phases for kinetics studies. On the other hand, an investigation of morphological changes in particle properties during growth is shown independent of agglomerated particle systems, such as crystal sizes, surface conditions, and agglomeration states (Gutsche et al. 2014; Gutsche et al. 2017). In detail, we show that the consumption of the crystalline zinc precursor can be recorded in a time-resolved fashion during the liquid-based synthesis by means of QPA and thus validated with gravimetric analysis proving a pseudo-first-order process kinetics of the overall AZO nanocrystal synthesis via the benzylamine route. Based on the obtained process kinetics, sampling time intervals are discussed in terms of optimal resolving the morphological changes of AZO during growth and the effort for further investigations can be reduced. In a further step, a comparison of the obtained process kinetics with TEM analysis leads to first conclusions about a generalized growth behavior of AZO nanocrystals during synthesis regarding morphological changes using a comparative growth factor. Furthermore, a more extensive timeresolved growth study of ongoing particle formation processes of AZO nanocrystals is carried out by simultaneously investigating morphological and fractal changes using SAXS analysis. Finally, using TEM, SAXS, and process kinetics data from the 
synthesis conditions obtained in this work, a new general growth model for AZO nanocrystals during synthesis via the benzylamine route is postulated getting an in-depth understanding of ongoing particle-based formation mechanisms at the nanoscale.

\section{Material and methods}

Synthesis and sample preparation of AZO nanocrystals

For the non-aqueous sol-gel synthesis of AZO nanocrystals via the benzylamine route, the crystalline precursors zinc acetylacetonate hydrate $\left(\mathrm{Zn}(\mathrm{acac})_{2}\right.$ powder, $\geq 99 \%$, Aldrich) and aluminum isopropoxide $\left(\mathrm{Al}(\mathrm{OiPr})_{3}, \geq 98 \%\right.$, Aldrich) at the initial concentration $C_{\mathrm{E}}=25 \mathrm{~g} \mathrm{~L}^{-1}$ were used, wherein the molar fraction of aluminum precursor was set to $2.5 \mathrm{~mol} \%$. The aromatic solvent benzylamine $\left(\mathrm{BnNH}_{2}\right.$, benzylamine for synthesis, $\geq 99 \%$, Merck) served as a reaction medium. To carry out the synthesis, a closed and ideally stirred steel reactor with glass inlet $(235 \mathrm{~mL})$ was used performing the process temperature $T_{\mathrm{R}}=110^{\circ} \mathrm{C}$ at a total overpressure of 1 barg. First, the sparingly soluble precursors were pre-dissolved in the reactor for $15 \mathrm{~min}$ at a temperature of $50^{\circ} \mathrm{C}$ by continuous stirring, before the reaction solution was quickly brought to process temperature with a heating rate of $30 \mathrm{~K} \mathrm{~min}^{-1}$. After reaching process temperature, the recording for reaction time was started. The sampling with a sample volume of $10 \mathrm{~mL}$ each took place at various reaction times by means of overpressure through a riser. The reaction process was terminated abruptly by rapid cooling to a temperature of $25^{\circ} \mathrm{C}$ in a water bath, due to the endothermic synthesis process. In case of sampling before the end of process, in addition to the reaction medium $\mathrm{BnNH}_{2}$, the obtained samples contained two crystalline phases AZO $\left|\mathrm{Zn}(\mathrm{acac})_{2}\right| \mathrm{BnNH}_{2}$, since complete precipitation of the unreacted zinc precursor occurred at room temperature.

With the exception of the QPA analysis method, further sample purification steps after sampling were necessary for analysis, in which the reaction medium $\mathrm{BnNH}_{2}$ and unreacted zinc precursor from the original sample AZO $\mid \mathrm{Zn}(\text { acac })_{2} \mid \mathrm{BnNH}_{2}$ had to be removed. On the one hand, the high boiling $\mathrm{BnNH}_{2}\left(T_{\mathrm{B}}=185^{\circ} \mathrm{C}\right)$ does not allow easy evaporation of the solvent needed to dry samples for gravimetric and TEM analysis. On the other hand, the unreacted crystalline precursors overlap the scattering signal of $A Z O$ for $q<2 \mathrm{~nm}^{-1}$ to be investigated by means of SAXS analysis. In a first purification step, the reaction medium $\mathrm{BnNH}_{2}$ was removed from the two crystalline phases $\mathrm{AZO} \mid \mathrm{Zn}(\mathrm{acac})_{2}$ by centrifugation for $15 \mathrm{~min}$ with a g-force of 7200 at $20^{\circ} \mathrm{C}$ (Centrifuge 5430R, Eppendorf). Subsequently, in a second step, the ethanol-soluble $\mathrm{Zn}(\mathrm{acac})_{2}$ precursor crystals were completely separated from the AZO nanocrystals by washing twice with ethanol excess of the factor 4 using centrifugation as described before. After the final washing step, the pure AZO nanocrystals, which were presented in an ethanol-based suspension, are well prepared for further SAXS, TEM, and gravimetric analyses.

\section{Characterization of $A Z O$ nanocrystals}

\section{SAXS}

For a comprehensive time-resolved characterization of particle formation processes via the benzylamine route, a non-invasive $\mathrm{X}$-ray analysis was performed using an in-house developed SAXS laboratory camera, which was described in detail elsewhere (Goertz et al. 2012; Guo et al. 2013a, b; Gutsche et al. 2014). The slitshaped X-ray beam, generated from a $1.2-\mathrm{kW}$ X-ray source, was monochromatized on $\mathrm{Cu}-\mathrm{K}-$ alpha $(\lambda=$ $0.154 \mathrm{~nm}$ ) and focused on the detection plane by means of a Goebel mirror and a collimation system. The samples were injected as a homogeneous suspension into a quartz glass capillary with a diameter of $1 \mathrm{~mm}$ and wall thickness $<40 \mu \mathrm{m}$. The X-ray scattering intensity $I(q)$ on samples was measured as a function of the scattering vector $q$ with $q=4 \pi / \lambda \cdot \sin (\theta / 2)$, wheras $\theta$ is the scattering angle and recorded by a two-dimensional hybrid photon counting X-ray detector with a pixel size of $0.172 \mathrm{~mm}$ (PILATUS 100K-S, DECTRIS) for $180 \mathrm{~s}$. In order to correct the scattering error of a slit-shaped beam, the background-corrected scattering data were desmeared by the direct method as described by Singh et al. (1993).

Structure analysis For the structural analysis by means of $S A X S$, ethanol-based suspensions of purified $A Z O$ nanocrystals, as described in the "Synthesis and sample preparation of AZO nanocrystals" section, were investigated. The scattering intensity of a monodisperse particle system, normalized to the squared classical electron 
radius $r_{e}^{2}$, including $N$ monodisperse particles with the single volume $V_{P}$ and the excess electron density $\Delta \rho_{e}$ in the exposed liquid volume $V$ is generally described in Eq. (1).

$I(q)=\frac{N}{V} \cdot \Delta \rho_{e}{ }^{2} \cdot V_{P}^{2} \cdot P(q) \cdot S(q)[a . u$.

Here the form factor $P(q)$ describes the intraparticle scatter fraction, whereas the interparticle scatter fraction is specified by the structure factor $S(q)$. For the description of particulate interactions for $q>0.1$, the influence of the structure factor with $S(q) \rightarrow 1$ can be neglected (Ashcroft and Lekner 1966).

The experimental determination of the radius of gyration $R_{g}$ from scattering data of an examined particle system with any geometry is carried out by the Guinier law in Eq. (2), which is valid for $q R_{q}<1.4$ in case of spherical particles (Glatter and Kratky 1982).

$I(q) \approx A \cdot \exp \left(-\frac{q^{2} R_{g}^{2}}{3}\right) \quad[$ a.u. $]$

Here, the exponential factor $A$ corresponds to the forward scattering $I(q=0)$. In the case of spherical particles, the geometric sphere diameter $d_{S}$ can be determined from $R_{g}$ using Eq. (3).

$d_{S}=2 \cdot \sqrt{\frac{5}{3}} \cdot R_{g} \quad[n m]$

From fractal analysis using $S A X S$, structural properties of fractal particle systems can be described by determining fractal dimensions $D_{f i}$. Based on scattering curves, fractal properties can be described using the power law in Eq. (4).

$I(q) \sim q^{-\nu}$

From Eq. (4), the fractal information (e.g. surface or mass fractals) can be extracted from the power $\nu$. In case of mass fractals, the mass fractal dimension $D_{f m}$ is described by Eq. (5) and for surface fractals Eq. (6) is to be used (Beaucage 1995; Boukari et al. 1997; Schmidt 1991), whereas the special case of $\nu=4$ for surface fractals leads to the Porod law, describing smooth surfaces with sharp density transition (Porod 1951).

$\nu=D_{f m}$ with $1<D_{f m}<3$
$\nu=6-D_{f s}$ with $2<D_{f s}<3$

Quantitative phase analysis The quantitative phase analysis (QPA) method can generally be used to identify and quantify crystalline phases independently of morphological states in crystalline particle systems. Using the SAXS laboratory camera, the X-ray intensity of phase $a$ in a multiphase mixture $I_{2 \theta}^{a}$ at a specific scattering angle $2 \theta$, as described in Eq. (7), could be detected occurring due to diffraction of X-rays with crystalline materials (Karlak and Burnett 1966).

$I_{2 \theta}^{a}=I_{0} \mathrm{~K}\left|F_{h k l}\right|^{2} \frac{1}{\mu^{*}} \frac{x^{a}}{\rho^{a}}$

$I_{0}$ describes the forward scattering $I(q=0)$, the constant $K$ combines all angle-dependent influences, the structure factor $F_{h k l}$ takes into account the scattering contribution of the crystal unit cell, the absorbance of the sample is described by the mean mass absorption coefficient of the multiphase mixture $\mu^{*}, \rho^{a}$ is the solid density of phase $a$, and $x^{a}$ describes the weight fraction of phase $a$.

\section{Gravimetry}

For the time-resolved kinetic analysis of the AZO nanocrystal growth during the synthesis, we used gravimetric analysis. As part of this analysis procedure, two sample purification steps, as described in the "Synthesis and sample preparation of AZO nanocrystals" section, were carried out after the sampling and the cooling process. Following from this, pure AZO nanocrystals present in ethanol were completely dried at $T=80^{\circ} \mathrm{C}$ for $24 \mathrm{~h}$ under ambient pressure in a drying oven ("UN260," Memmert). Here, the solid dry matter concentration of the AZO crystals $C_{\mathrm{AzO}}$ was investigated with an analytical scale (Secura 224-1S, Sartorius) by using Eq. (8), dependent on sampling volume $V_{\mathrm{s}}$ and dry matter $m_{\mathrm{AZO}}$.

$C_{\mathrm{AZO}}=\frac{m_{\mathrm{AZO}}}{V_{\mathrm{s}}}\left[\mathrm{g} \mathrm{L}^{-1}\right]$

\section{TEM}

To characterize size, shape, and crystal structure at selected growth states of AZO nanocrystals during synthesis via benzylamine route, a TEM (CM12, Philipps) 
was used. For this, the samples were diluted with ethanol and air-dried on a TEM grid at $25^{\circ} \mathrm{C}$. The mean size distribution of AZO was evaluated by investigating at least 900 particles using the software ImageJ.

Kinetic model describing AZO nanocrystal synthesis

For a time-resolved description of consumption and growth processes during the AZO synthesis via the benzylamine route, the pseudo-first-order kinetics model was used. Similar first-order kinetics have been reported before for the non-aqueous synthesis of $\mathrm{TiO}_{2}$ nanoparticles (Garnweitner and Grote 2009). For the purposes of applying this model to the AZO process, the following assumptions have been made for simplicity: Firstly, due to the low Al precursor concentration $(2.5 \mathrm{~mol}-\%)$ compared to the zinc precursor, its participation in the reaction process was neglected, thus $C_{\text {Educt }}$ $\approx C_{\mathrm{Zn}(\mathrm{acac})_{2}}$ applies for the educt concentration. Secondly, the formation of undesired by-products was excluded and the molar ratio of 1 was assumed for $\mathrm{Zn}(\mathrm{acac})_{2}$ and AZO with respect to the reaction conversion. Consequently, a complete reaction sequence from the work of Pinna et al. (2005) is described in Eq. (9), in which the benzyl group was abbreviated to $\mathrm{Bn}$.

$$
\begin{array}{ll} 
& 1 \cdot \mathrm{AZO} \\
4 \cdot \mathrm{Bn}-\mathrm{NH}_{2} \rightarrow \rightarrow & +2 \cdot \mathrm{Bn}-\mathrm{N}=\mathrm{C}\left(\mathrm{CH}_{3}\right)_{2} \\
+1 \cdot \mathrm{Zn}(\mathrm{acac})_{2} \quad & +2 \cdot \mathrm{Bn}-\mathrm{NH}-\mathrm{C}(=\mathrm{O}) \mathrm{CH}_{3} \\
& +1 \cdot \mathrm{H}_{2} \mathrm{O}
\end{array}
$$

From the potency approach of the reaction rate, an expression of the AZO formation rate $r_{\mathrm{AZO}}$, depending on the unreacted $\mathrm{Zn}(\mathrm{acac})_{2}$ concentration $C_{\mathrm{Zn}(\mathrm{acac})_{2}}(t)$ and the rate constant $k\left[\mathrm{~s}^{-1}\right]$, was obtained in Eq. (10).

$r_{\mathrm{AZO}}(t)=-\frac{d C_{\mathrm{Zn}(\mathrm{acac})_{2}}}{d t}=k C_{\mathrm{Zn}(\mathrm{acac})_{2}}(t)$

After separation of variables, integration, and exponentiation, a pseudo-first-order model resulted in Eq. (11), describing a time-dependent exponential consumption of $C_{\mathrm{Zn}(\mathrm{acac})_{2}}(t)$ as a function of initial zinc precursor concentration $C_{\mathrm{Zn}(\mathrm{acac})_{2}}(0)$.

$C_{\mathrm{Zn}(\text { acac })_{2}}(t)=C_{\mathrm{Zn}(\text { acac })_{2}}(0) e^{-k t}$

In case of full conversion, for the final product concentration $C_{\mathrm{AZO}}(\infty)=C_{\mathrm{Zn}(\mathrm{acac})_{2}}(0)$ applies, and with the expression: $C_{\mathrm{AZO}}(t)=C_{\mathrm{Zn}(\mathrm{acac})_{2}}(0)-C_{\mathrm{Zn}(\mathrm{acac})_{2}}(t)$, the pseudo-first-order model of restricted exponential growth of AZO concentration $C_{\mathrm{AZO}}(t)$ resulted in Eq. (12) with equal rate constant.

$$
C_{\mathrm{AZO}}(t)=C_{\mathrm{AZO}}(\infty)\left(1-e^{-k t}\right)
$$

\section{Results and discussions}

Kinetics of AZO nanocrystal synthesis using QPA

In this work, the QPA technique for the time-resolved study of zinc precursor consumption during the AZO synthesis process was used to gain insights into the kinetics of particle formation processes. However, it was challenging to prepare the samples of the AZO synthesis process for QPA measurement technique. Here, a new method for sample preparation and analysis has been developed, despite the high corrosivity of the solvent benzylamine, allowing a timely analysis of the particle system after synthesis without major interventions (e.g., solvent exchange). To avoid corrosive damage to the measuring system, the samples had to be investigated in a corrosion-resistant capillary exclusively at room temperature for a maximum dwell of $3 \mathrm{~min}$. Cooling to room temperature provides two important advantages that could be exploited for the measurement process by means of QPA. On the one hand, the endothermic reaction process was stopped to prevent an uncontrolled change of the particle system during the measuring process. On the other hand, the insolubility of the zinc precursor at room temperature could be exploited forcing crystalline precipitation of the unreacted zinc precursor so that detection by QPA could be generally enabled. Since a large part of the quantities from Eq. (7) in the "Characterization of AZO nanocrystals" section are empirically difficult to access, a theoretical prediction of mass fractions based on measured scattering curves is only possible to a limited extent. Therefore, in our specific case, a calibration of our SAXS laboratory camera was necessary describing the linear relationship between $I_{h k l}^{a}$ and $x^{a}$ from Eq. (7) for the precursor material $\mathrm{Zn}(\mathrm{acac})_{2}$ in the reference system $\mathrm{Zn}(\mathrm{acac})_{2} \mid \mathrm{BnNH}_{2}$. For this purpose, the scattering intensities of $\mathrm{Zn}(\mathrm{acac})_{2} \mid \mathrm{BnNH}_{2}$ reference samples in the concentration range $6.13 \mathrm{~g} \mathrm{~L}^{-1} \leq C_{\mathrm{Zn}(\mathrm{acac})_{2}} \leq$ $17,65 \mathrm{~g} \mathrm{~L}^{-1}$ were recorded using the SAXS camera. Higher concentrations could not be considered due to 
the rapidly increasing viscosity of the samples. Bragg reflections of crystalline $\mathrm{Zn}(\mathrm{acac})_{2}$ lattice planes in the scattering angle range $2 \theta=13^{\circ}-17^{\circ}$ could be observed, as shown in Fig. 1a. Therein, the scattering intensity decreases with lower $\mathrm{Zn}(\mathrm{acac})_{2}$ concentrations until finally the background scattering of pure solvent $\mathrm{BnNH}_{2}$ is achieved. Thus, the observed Bragg reflexes within the range $13^{\circ} \leq$ $2 \theta \leq 17^{\circ}$ could be clearly assigned to the zinc precursor.

Assigning scattering peak intensities of the dominant reflection after background scattering deduction of pure $\mathrm{BnNH}_{2}$ at $2 \theta=16^{\circ}$ to concentrations of the reference suspensions resulted in a linear correlation between

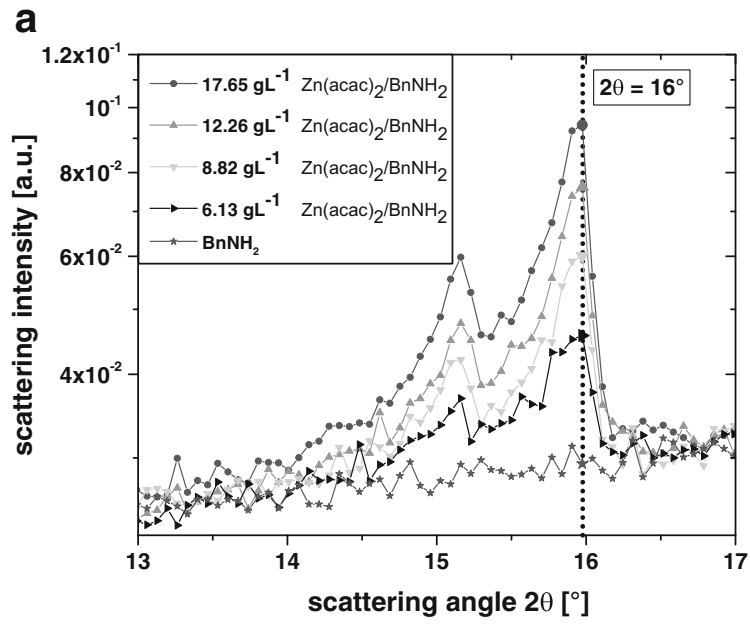

b

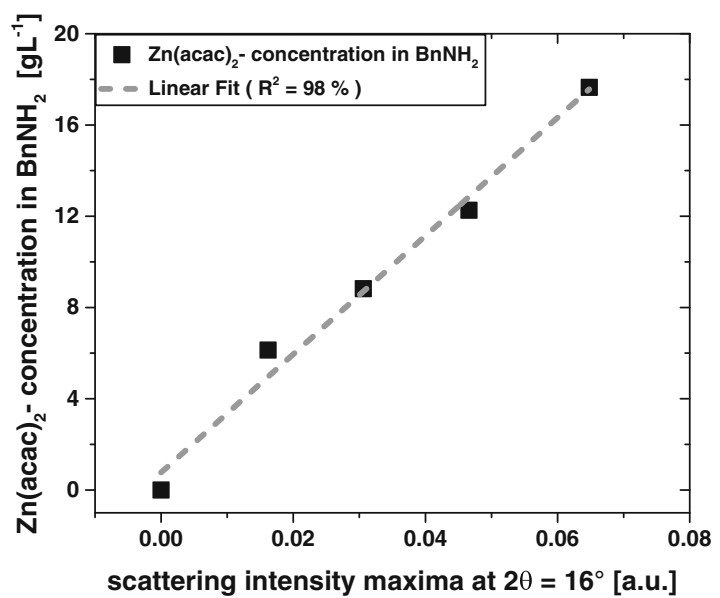

Fig. 1 Decreasing Bragg reflections of crystalline $\mathrm{Zn}(\mathrm{acac})_{2}$ with decreasing concentration in $\mathrm{BnNH}_{2}$ in the range $13^{\circ} \leq 2 \theta \leq$ $17^{\circ}$ (a) and the associated linear approximated (dashed line) intensity maxima after background scattering deduction at $2 \Theta=16^{\circ}$ (black squares) plotted over the correlated $\mathrm{Zn}(\mathrm{acac})_{2}$ concentration (b)
$\mathrm{Zn}(\mathrm{acac})_{2}$ concentration and scattering intensity with a very high coefficient of determination $\left(R^{2}=98 \%\right)$, which is shown in Fig. 1b. Based on the hereby obtained linear relationship, which is also confirmed in Eq. (7), the general validity of our SAXS camera for the quantitative detection of the zinc precursor in pure $\mathrm{BnNH}_{2}$ could thus be confirmed. Based on the linear regression, a linear calibration function for the quantitative investigation of $\mathrm{Zn}(\mathrm{acac})_{2}$ concentrations $C_{\mathrm{Zn}(\mathrm{acac})_{2}}$ for the crystalline system $\mathrm{Zn}(\mathrm{acac})_{2} \mid \mathrm{BnNH}_{2}$ could be obtained in Eq. (13). Based on works of Zellmer et al. (2015), Soofivand et al. (2014), Kelchtermans et al. (2013), and Pinna et al. (2005), an assumption was made that the scattering reflections of crystalline AZO $\left(2 \theta>30^{\circ}\right)$ do not affect the reflections of $\mathrm{Zn}(\mathrm{acac})_{2}$ at $13^{\circ} \leq 2 \theta \leq 17^{\circ}$ obtained here. Thus, Eq. (13) also remains valid for the investigated system containing two crystalline phases $\mathrm{AZO}\left|\mathrm{Zn}(\mathrm{acac})_{2}\right| \mathrm{BnNH}_{2}$.

$C_{\mathrm{Zn}(\mathrm{acac})_{2}}=(259 \pm 7 \%) \cdot \mathrm{I}_{2 \theta=16^{\circ}}^{\mathrm{Zn}(\mathrm{acac})_{2}}\left[\mathrm{~g} \mathrm{~L}^{-1}\right]$

To validate the applicability of the QPA method in terms of time-resolved analysis of $\mathrm{Zn}(\mathrm{acac})_{2}$ consumption, an AZO synthesis was carried out at reaction temperature $T_{\mathrm{R}}=110^{\circ} \mathrm{C}$ and an initial precursor concentration $C_{\mathrm{E}}=25 \mathrm{~g} \mathrm{~L}^{-1}$. In Fig. 2, correlated $\mathrm{Zn}(\mathrm{acac})_{2}$ concentrations of multiphase reaction samples AZO | $\mathrm{Zn}(\mathrm{acac})_{2} \mid \mathrm{BnNH}_{2}$, determined from the evaluation of the dominant reflection peak at $2 \theta=16^{\circ}$ by QPA and using the calibration function from Eq. (13), are plotted over reaction time (black squares).

The correlated zinc precursor concentration $C_{\mathrm{Zn}(\text { acac })_{2}}$ $(t)$ in Fig. 2 decreases exponentially with increasing reaction time in the period $0<t_{\mathrm{R}}<100$ min up to full consumption of $\mathrm{Zn}(\mathrm{acac})_{2}$. For a quantitative description of the consumption processes of the zinc precursor during the AZO synthesis via the benzylamine route, the pseudo-first-order kinetics model from Eq. (11) has been fitted to the QPA data in Fig. 2 by a high coefficient of determination $\left(R_{\mathrm{Zn}(\mathrm{acac})_{2}}^{2} \approx 98 \%\right)$. Due to this fact, the determination of important process parameters of the $\mathrm{Zn}(\mathrm{acac})_{2}$ consumption from Eq. (11) was feasible, such as the rate constant $k_{\mathrm{Zn}(\mathrm{acac})_{2}}^{110^{\circ} \mathrm{C}} \approx 11.3 \cdot 10^{-4} \mathrm{~s}^{-1} \pm 15 \%$, an extrapolated initial $\mathrm{Zn}(\mathrm{acac})_{2}$ concentration $C_{\mathrm{Zn}(\mathrm{acac})_{2}}(0) \approx 23.8 \mathrm{~g} \mathrm{~L}^{-1} \pm 3 \%$, and an expected duration of the reaction $\left.\left.t_{\mathrm{Zn}\left(10^{\circ} \mathrm{C}\right.}\right)_{2}\right)_{2}(\infty) \approx 52 \mathrm{~min} \pm \ll 1 \%$, after which $97 \%$ of $\mathrm{Zn}(\mathrm{acac})_{2}$ were consumed. In order to validate the pseudo-first-order kinetics for the overall 


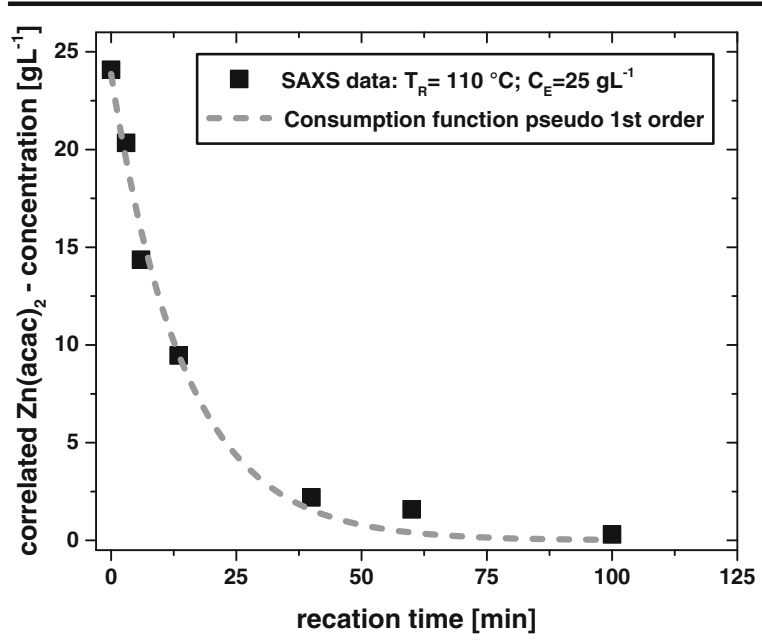

Fig. 2 Correlated $\mathrm{Zn}(\mathrm{acac})_{2}$ concentration dependent on reaction time from QPA by means of SAXS data after using Eq. (13) (black squares) at constant process conditions $\left(T_{\mathrm{R}}=110^{\circ} C ; C_{\mathrm{E}}=\right.$ $25 \mathrm{~g} \mathrm{~L}^{-1}$ ) and the approximated pseudo-first-order consumption kinetic function based on Eq. (11) with $R^{2} \approx 98 \%$ (dashed line)

synthesis process, the growth of the AZO nanocrystals by gravimetry was additionally investigated. A QPA of the crystalline AZO reflections was not feasible due to the limited angular resolution of our SAXS laboratory camera $\left(0^{\circ}<2 \Theta<30^{\circ}\right)$. In this context, the previously determined normalized pseudo-first-order consumption model function of $\mathrm{Zn}(\mathrm{acac})_{2}$, based on QPA, and the associated normalized pseudo-first-order growth model function of AZO from Eq. (12), based on gravimetric data with a very high coefficient of determination $\left(R_{\mathrm{AZO}}^{2} \approx 99 \%\right.$ ), are plotted in Fig. 3 .

Here, the qualitative trajectories of the respective growth and consumption functions formed an intersection at a concentration ratio of approx. 50\%, indicating pseudo-first-order kinetics for the overall synthesis of AZO nanocrystals via the benzylamine route. The final proof could be provided by the comparison of the consumption and growth rate constants in Fig. 3 by calculating their relative mean deviation $\overline{\Delta k}_{\mathrm{AZO} / \mathrm{Zn}(\mathrm{acac})_{2}}=13 \%$. By comparing $\overline{\Delta k}_{\mathrm{AZO} / \mathrm{Zn}(\mathrm{acac})_{2}}$ of $\mathrm{AZO}$ and $\mathrm{Zn}(\mathrm{acac})_{2}$ kinetics with the occurring model-related errors of $k$ in Fig. 3, obviously no difference can be established between the two rate constants of each respective process. Hence, it was generally proven that both the consumption kinetics of $\mathrm{Zn}(\mathrm{acac})_{2}$ and the growth kinetics of AZO are identical at the investigated process conditions. This fact thus proved that the synthesis of AZO nanocrystals via the benzylamine route follows generally a pseudo-first- order kinetics, as originally assumed in the "Kinetic model describing AZO nanocrystal synthesis" section. Furthermore, it could be shown that the process duration compared to the work of Zellmer et al. (2015) from $\sim 30 \mathrm{~min}$ at $T_{\mathrm{R}}=200^{\circ} \mathrm{C}$ to $\sim 60 \mathrm{~min}$ at $T_{\mathrm{R}}=110^{\circ} \mathrm{C}$ could be significantly extended and thus a better time resolution of the particle formation processes was achieved for further investigations.

Growth behavior of AZO using kinetic model and TEM

For a precise investigation of the growth behavior of AZO nanocrystals during the synthesis via the benzylamine route, knowledge of its growth kinetics offers decisive advantages. The previously obtained pseudo-first-order kinetic model contains important information regarding the time of sampling and subsequent morphological investigations obtaining an optimal description of the time-resolved growth behavior of AZO nanocrystals. Based on the previously obtained kinetic parameters, a region with high rates of change in crystal growth in the time period of $0 \mathrm{~min} \leq t \leq$ 60 min could be defined, in which a high sampling rate was performed with increasing time intervals, whereas only two more sampling times $t=120 \mathrm{~min}, t=135 \mathrm{~min}$ were necessary to resolve the final state of AZO growth (see Table 1). It was thus possible to ensure the optimum description of the entire AZO nanocrystals growth course with as few sampling steps as possible, on the one hand with a high resolution of expected changes in crystal growth and on the other hand to ensure the complete acquisition of crystal growth by detecting the expected plateau.

In addition to the determination of the optimum sampling intervals, predictions on the growth of single particles could be made using the parameters from the kinetics model function in the "Kinetics of AZO nanocrystal synthesis using QPA" section. Taking into account some assumptions, e.g., finished nucleation processes before sampling and consequently a constant particle number concentration $\left(C_{n}(t)=C_{n}=\right.$ const. $)$, a monodisperse growth of spherical particles with constant density ( $\rho=$ const.) and neglecting particleparticle interactions, it can be concluded from the increase of the total particle mass concentration to an equivalent increase in the particle diameter of a spherical single particle. Here, the total mass concentration $C_{\mathrm{AZO}}(t)$ of the entire AZO particle collective consisting of monodisperse particles with the previously assumed 
Fig. 3 Normalized pseudo-firstorder $\mathrm{Zn}(\mathrm{acac})_{2}$ consumption model functions based on QPA data by SAXS and associated normalized pseudo-first-order AZO growth model functions from gravimetric data at constant process conditions: $T_{\mathrm{R}}=80^{\circ} \mathrm{C}$; $C_{\mathrm{E}}=12.5 \mathrm{~g} \mathrm{~L}^{-1}$

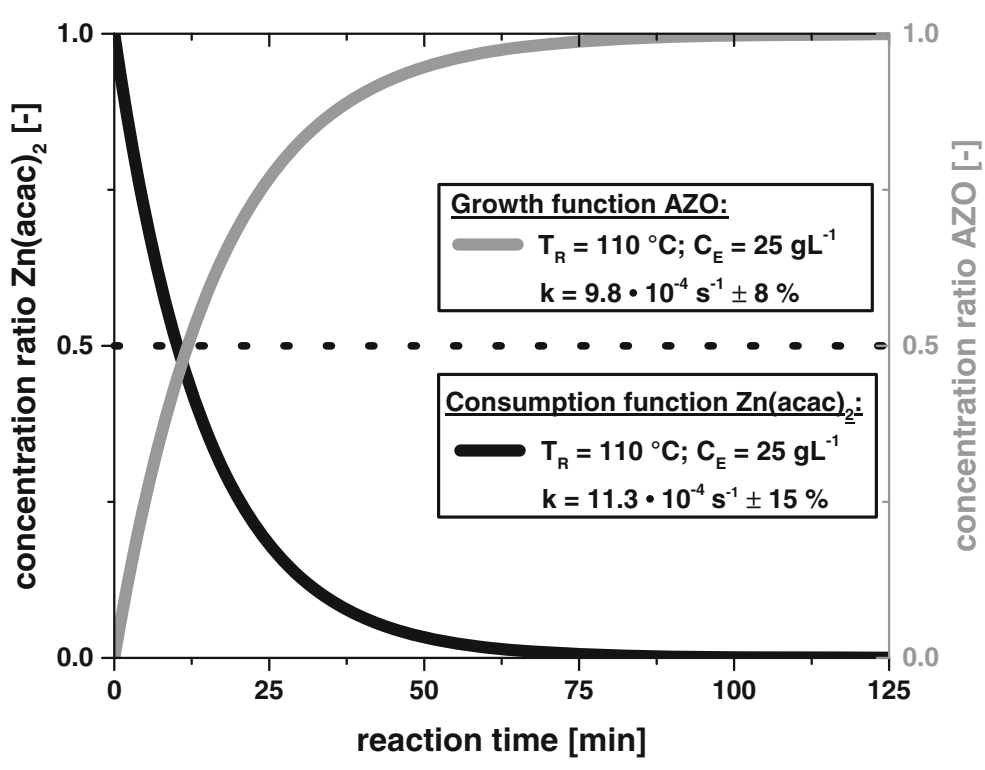

constant number concentration $C_{n}$ and spherical diameter $d_{\mathrm{S}}(t)$ at reaction time $t$ can be calculated with Eq. (14).

$C_{\mathrm{AZO}}(t)=C_{n} \cdot \rho \frac{\pi \cdot d_{\mathrm{S}}^{3}(t)}{6}\left[\mathrm{~g} \mathrm{~L}^{-1}\right]$

Thus, combining Eq. (14) for two different time steps at the earliest detectable $t_{\min }$ and at the final reaction time $t_{\infty}$ results in Eq. (15), calculating a maximum global growth ratio $X^{i}$ of the synthesis process.

$X^{i}=\frac{d_{\mathrm{S}}\left(t_{\infty}\right)}{d_{\mathrm{S}}\left(t_{\min }\right)}=\sqrt[3]{\frac{C\left(t_{\infty}\right)}{C\left(t_{\min }\right)}}[-]$

The obtained maximum growth ratio in Eq. (15) is a general tool comparing the growth progress between different measurement methods, based on the detection of the particle size on the one hand and the detection of the mass concentration on the other. Extracting the already known mass concentrations from our pseudofirst-order kinetics model of $A Z O$ nanocrystals both at

Table 1 Optimized times of sampling after start of reaction process at constant process conditions $\left(T_{\mathrm{R}}=110^{\circ} \mathrm{C} ; C_{\mathrm{E}}=25 \mathrm{~g} \mathrm{~L}^{-1}\right)$ based on pseudo-first-order kinetics model function in the "Kinetics of AZO nanocrystal synthesis using QPA" section

Times of sampling after start of reaction [min]

\begin{tabular}{llll}
\hline 3 & 13.5 & 42 & 120 \\
6 & 20 & 60 & 135 \\
\hline
\end{tabular}

the earliest stage of growth $C\left(t_{\min }=3 \mathrm{~min}\right)=1.1 \mathrm{~g} \mathrm{~L}^{-1} \pm$ $5 \%$ and at the end of growth $C\left(t_{\infty}=135 \mathrm{~min}\right) \approx$ $C_{\mathrm{AzO}}(\infty)=7.3 \mathrm{~g} \mathrm{~L}^{-1} \pm 1 \%$, the determination of the maximum expected growth ratio $X^{\text {Kin. }} \approx 2$ of the $\mathrm{AZO}$ growth process was possible using Eq. (15). Based on $X^{\text {Kin. }}$, it is thus possible to deduce the maximum expected change in primary particle size if an ideal growth of monodisperse single particles is assumed. To verify the previously assumed growth behavior of the considered process, TEM images of AZO nanocrystals, synthesized under the previously introduced constant process conditions, sampled after reaction times of $t=3 \mathrm{~min}$ and $t=$ $135 \mathrm{~min}$ on the basis of Table 1 are shown in Fig. 4.

The particle size analysis of these TEM images showed an increase of the mean maximum Feret diameter from $\bar{d}_{\mathrm{F}, \max }(t=3 \mathrm{~min}) \approx 11.7 \mathrm{~nm} \pm 25 \%$ to $\bar{d}_{\mathrm{F}, \text { max }}(t=135 \mathrm{~min})=\bar{d}_{\mathrm{F} \text {, } \max }\left(t_{\infty}\right) \approx 72.0 \mathrm{~nm} \pm 10 \%$.

Here, a crystal growth was observed by the increase of the maximum Feret diameter during the considered period. Due to the assumption of spherical crystals, $\bar{d}_{\mathrm{F}, \max }=d_{S}$ was supposed. Based on TEM analysis a growth ratio $X^{\mathrm{TEM}} \approx 6$ was obtained using Eq. (15).

The direct comparison between the previously described TEM- and kinetic-based growth analysis methods in Table 2 shows a clear difference in the maximum growth ratio of a factor of three, which could not be attributed to measurement errors due to the order of magnitude.

Consequently, it can be seen that the AZO nanocrystals actually grew to significantly larger 
Fig. 4 TEM images of AZO nanocrystals synthesized at constant process conditions $\left(T_{\mathrm{R}}=\right.$ $110^{\circ} \mathrm{C} ; C_{\mathrm{E}}=25 \mathrm{~g} \mathrm{~L}^{-1}$ ) and sampled at an early stage of growth after $t=3 \mathrm{~min}$ (a) and at the end of growth after $t=$ $135 \mathrm{~min}$ (b). Hexagonal-shaped nanocrystals are marked with arrows and dashed lines (b)
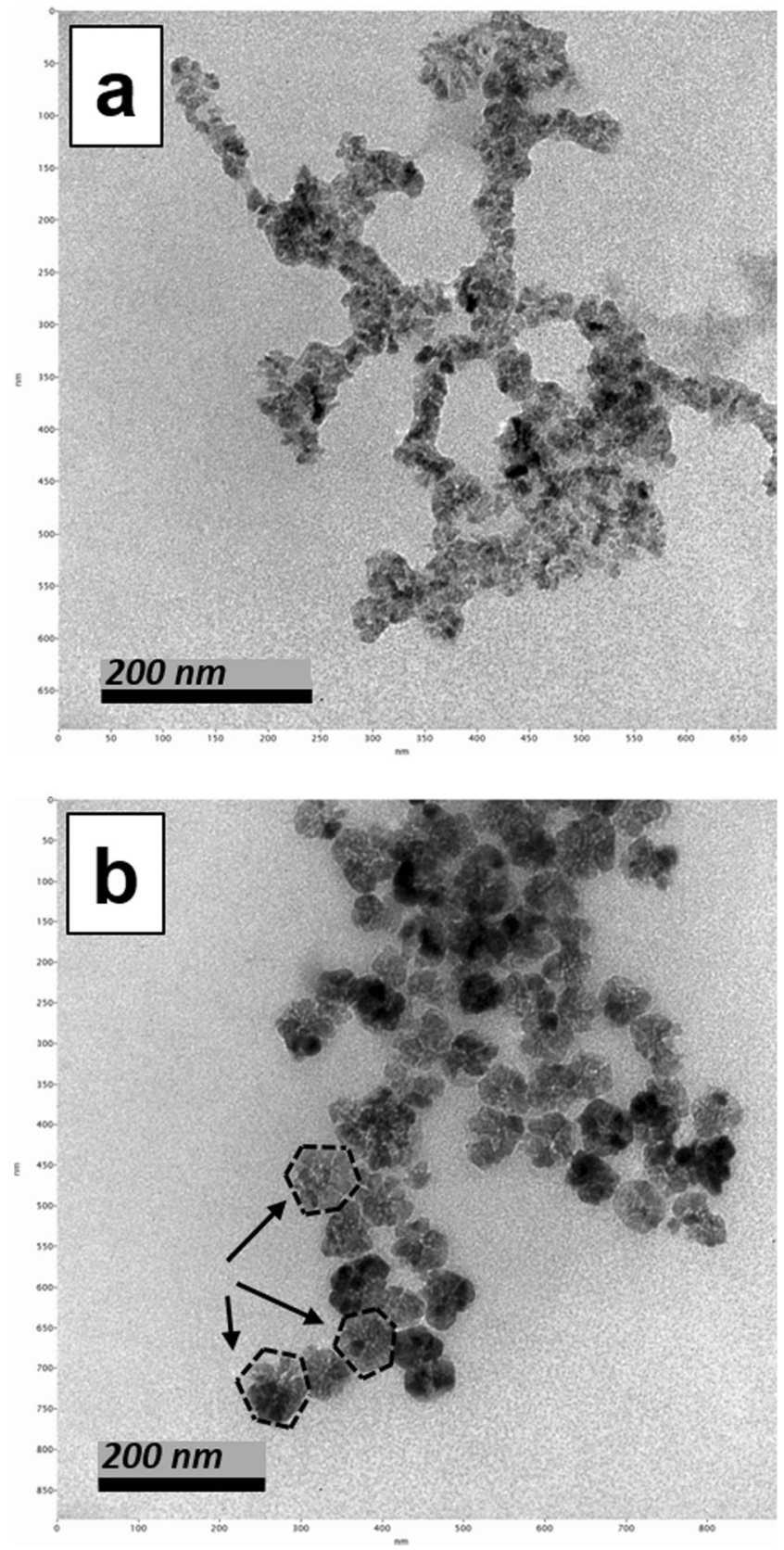

crystals during their growth process (see Fig. 4b), as originally predicted by the kinetics model function. Based on this fact, it could be suggested that the growth of the AZO nanocrystals via the benzylamine route did not result in pure single crystals from a classical crystallization mechanism but in mesocrystal structures by a non-classical crystallization mechanism (Cölfen and Antonietti 2005; Ludi et al. 2012; Niederberger and Cölfen 2006; Zhang et al. 2009), which is shown in detail in Fig. 5 by examining a single hexagonalshaped particle from Fig. 4b.

With detailed observation of the gray value trend along the dashed line drawn in Fig. 5a at the cross section of a single particle, in Fig. 5b, an internal grain boundary (lower peak, marked with the lower dashed horizontal line) could be observed between the two significant phase boundaries of the examined single particle (two dominant peaks, marked with the upper 
Table 2 Comparison of two maximum growth ratios of AZO between TEM data $\left(X^{\mathrm{TEM}}\right)$ and kinetic model data $\left(X^{\mathrm{Kin}}\right)$ including the respective raw data at the earliest stage of growth $(t=3 \mathrm{~min})$ and at the end of growth $(t=135 \mathrm{~min})$

\begin{tabular}{lccc}
\hline Method & $t=3$ min & $t=135$ min & Max. growth ratio $X^{i}[-]$ \\
\hline TEM data: & $11.7 \pm 25 \%$ & $72 \pm 10 \%$ & 6 \\
$\begin{array}{l}d_{\text {S, TEM }[\mathrm{nm}]} \\
\text { Kinetics model data: }\end{array}$ g L $\left.^{-1}\right]$ & $1.1 \pm 5 \%$ & $7.3 \pm 1 \%$ & 2 \\
\hline
\end{tabular}

dashed horizontal line). Consequently, the internal grain boundaries strongly indicated that the AZO nanocrystals has been formed aggregates during the stages of growth and grew consequently into mesocrystal structures in the

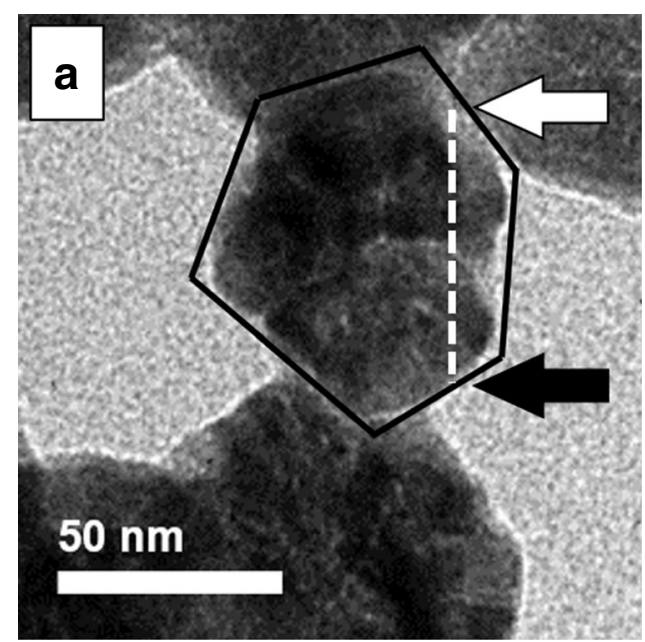

b

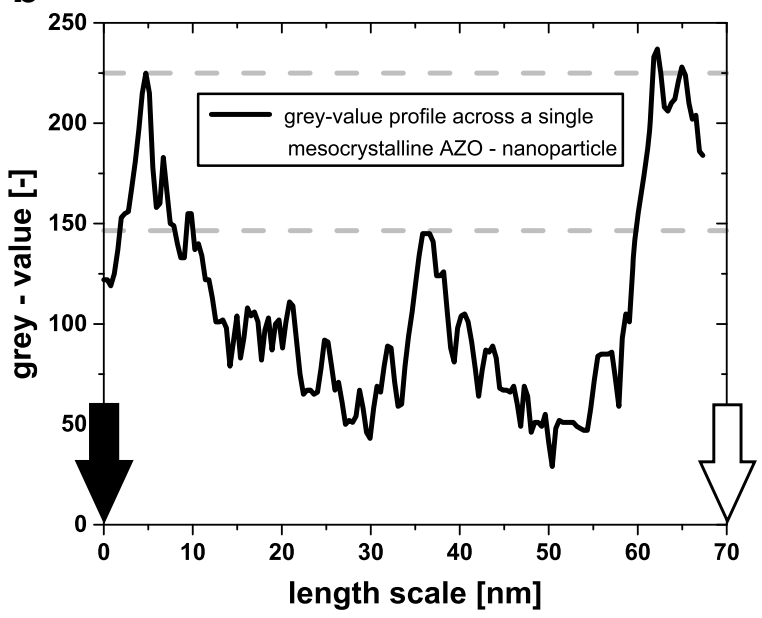

Fig. 5 Detailed observation of a hexagonal-shaped (solid line) single polycrystalline AZO nanoparticle, synthesized at constant process conditions $\left(T_{\mathrm{R}}=110^{\circ} \mathrm{C} ; C_{\mathrm{E}}=25 \mathrm{~g} \mathrm{~L}^{-1}\right)$ and sampled after end of growth (a) and its respective gray value profile plotted over the length scale of the drawn dashed line (b) further growth process until internal grain boundaries remained (Cölfen and Antonietti 2005). Additionally, a typical hexagonal shape of the AZO mesocrystals, which was highlighted on the one hand by arrows and dashed lines in Fig. $4 \mathrm{~b}$ and on the other by a solid line in Fig. 5a, led to the presumption of an oriented aggregation process during growth, which was described elsewhere (Jia and Gao 2008; Ludi et al. 2012; Penn and Soltis 2014). Moreover, the observed hexagonal shape of the AZO mesocrystals closely resembled the hexagonal wurtzite lattice framework of a classical zinc oxide single crystal (Wang 2004). In turn, it has shown that a hexagonal shape represents an energetically favorable arrangement of AZO primary crystals. In contrast to a complete fusion of primary crystals, as reported in the works of Olliges-Stadler et al. (2013) and Jia and Gao (2008), it was assumed here that the surface wetting of the organic solvent leads to the stabilization of the primary crystals and thus results in AZO mesocrystals with remaining internal grain boundaries, as reported from Song and Cölfen (2010).

Morphological analysis of crystal growth using SAXS

In order to describe the growth behavior of the previously investigated AZO nanocrystals $\left(T_{\mathrm{R}}=110^{\circ} \mathrm{C}\right.$; $C_{\text {prec }}=25 \mathrm{~g} \mathrm{~L}^{-1}$ ) in more detail in terms of time resolution, the ethanol-based samples of AZO were examined time-resolved in the range $3 \mathrm{~min} \leq t \leq 135 \mathrm{~min}$ by means of our SAXS laboratory camera. The resulting background purified scattering intensities $I$ were plotted twice logarithmically as functions of the scattering vector $q<1 \mathrm{~nm}^{-1}$ and the reaction time $t$ in Fig. 6 (dots).

Based on the highlighted maximum scattering intensities $I\left(q_{\mathrm{min}}\right)$ of each curve at constant minimum scattered angle $q_{\min }=0.108 \mathrm{~nm}^{-1}$ (see values of enlarged dots in Fig. 6), an increase in total scattering intensity $I(q)$ can be seen with increasing reaction progress. Since $I(q)$ is square linked in Eq. (1) to the particle 
volume of a single particle $V_{\mathrm{P}}$, a growth of nanocrystals could be generally derived from the time-dependent increase of $I(q)$, considering the assumption of finished nucleation before starting growth.

Moreover, the model function of Guinier from Eq. (2) was fitted to the respective scattering curves in Fig. 6 with $R^{2}>95 \%$ in terms of a time-resolved investigation of crystal size (see solid lines with gray filling). For a detailed evaluation of the time dependency of the Guinier model, the appropriate fits from Fig. 6 were again examined on the basis of a 2D plot in Fig. 7a.

In addition to the previously discussed timedependent increase of $I(q)$, a continuous increase in scattering intensity and a left shift of the obtained Guinier fits towards smaller scattering vectors were observed with increasing $t$. This shift showed an increase in $R_{g}$ with reaction time, because of the direct reciprocal relation of $R_{g}$ and $q$ in Eq. (2). Based on $R_{g}$, the correlated $d_{s}$ was calculated for every time step using Eq. (3) and subsequently plotted over $t$ in Fig. 7b. Here, a limited increase of $d_{s}$ from $14.2 \mathrm{~nm} \pm 17 \%$ up to $32.5 \mathrm{~nm} \pm 3 \%$ could be observed in the investigated range of $3 \mathrm{~min} \leq t \leq 135 \mathrm{~min}$, whereas the qualitative course of crystal growth could be successfully verified by the similarity to the previously obtained pseudo-firstorder kinetic model from the "Kinetics of AZO nanocrystal synthesis using QPA" section and due to the very high coefficient of determination $\left(R^{2}>98 \%\right)$.

To first clarify the question of which type of particle sizes were detected by means of SAXS, primary crystal size or size of agglomerates, a comparison was made with TEM images from the "Growth behavior of AZO using kinetic model and TEM" section Table 3 compares the mean particle sizes $d_{s}$ determined by means of TEM (see Fig. 4) and SAXS (see Fig. 7b) at the earliest sampling time $t=3 \mathrm{~min} \min$ and at the end of the crystal growth $t=135 \mathrm{~min}$ with the corresponding maximum growth ratios based on Eq. (15).

After a growth of $t=3 \mathrm{~min}$, both the average particle sizes determined by means of TEM and SAXS were in the same order of magnitude. Thus, the evidence of the measurability of mean primary crystal sizes by means of SAXS in the early growth stage was confirmed by comparison with TEM in Fig. 4a. In contrast, the final mean crystal size after $t=135$ min determined by TEM was almost more than twice as high than by SAXS, which becomes particularly clear with regard to the difference of the appropriate maximum growth ratios $X^{\text {SAXS }}$ and $X^{\text {TEM }}$ in Table 3. In addition, when comparing the SAXS data with the kinetic model data from the "Kinetics of AZO nanocrystal synthesis using QPA" section, which are also listed in Table 3, the equality of $X^{\text {SAXS }}$ and $X^{\text {Kin. }}$ could be observed, indicating an ideal growth behavior of single monodisperse AZO crystals without formation of mesocrystals was recorded by means of SAXS. Based on the two comparing facts regarding SAXS data: the similarity of $d_{\mathrm{S}}$, TEM and $d_{\mathrm{S}}$, SAXS at $t=3$ min and the equality of $X^{\mathrm{SAXS}}$ and $X^{\text {Kin. }}$ in Table 3, it was proven that the growth behavior of AZO primary crystals could be detected by means of SAXS, while the mesocrystals could not be resolved due to resolution limits of the used SAXS laboratory camera. In this section, it has been shown that the SAXS measuring technique is able to study the growth of the primary particles despite its agglomeration behavior and thus be contributed to a comprehensive understanding of the growth behavior of AZO mesocrystals during synthesis via the benzylamine route.

Fractal analysis during AZO growth using SAXS

In addition to the primary particle size, additional information about the fractal properties of the AZO nanocrystals could be obtained during synthesis from the SAXS data in Fig. 6. In order to investigate fractal properties of AZO in detail, the scattering intensity $I(q)$ of AZO at $t=135 \mathrm{~min}$ was plotted double logarithmically over $q$ in Fig. 8 including the respective Guinier model fit (dashed line), which was previously discussed in the "Morphological analysis of crystal growth using SAXS" section.

To determine the fractal dimension of the mass and surface fractals of the previously investigated AZO primary crystals, the power law from the Porod model in Eq. (4) was approximated twice to the respective scattering curve in Fig. 8 as straight lines from whose gradients the respective fractal dimensions were obtained. In detail, the change in the slope of the total scattering curve in the surrounding area of the Guinier plot (dashed line), which could be specified with $q^{*}(t=135 \mathrm{~min}) \approx$ $0.18 \mathrm{~nm}^{-1}$ at the intersection of both power law plots (solid lines), was analyzed. In a first step, the mass fractal dimension $D_{\mathrm{fm}}(t=135 \mathrm{~min}) \rightarrow 3$ could be determined from the slope of the power law for $q$ $<q^{*}$ in Fig. 8. The detection of mass fractals generally confirms the fact that the studied AZO primary 


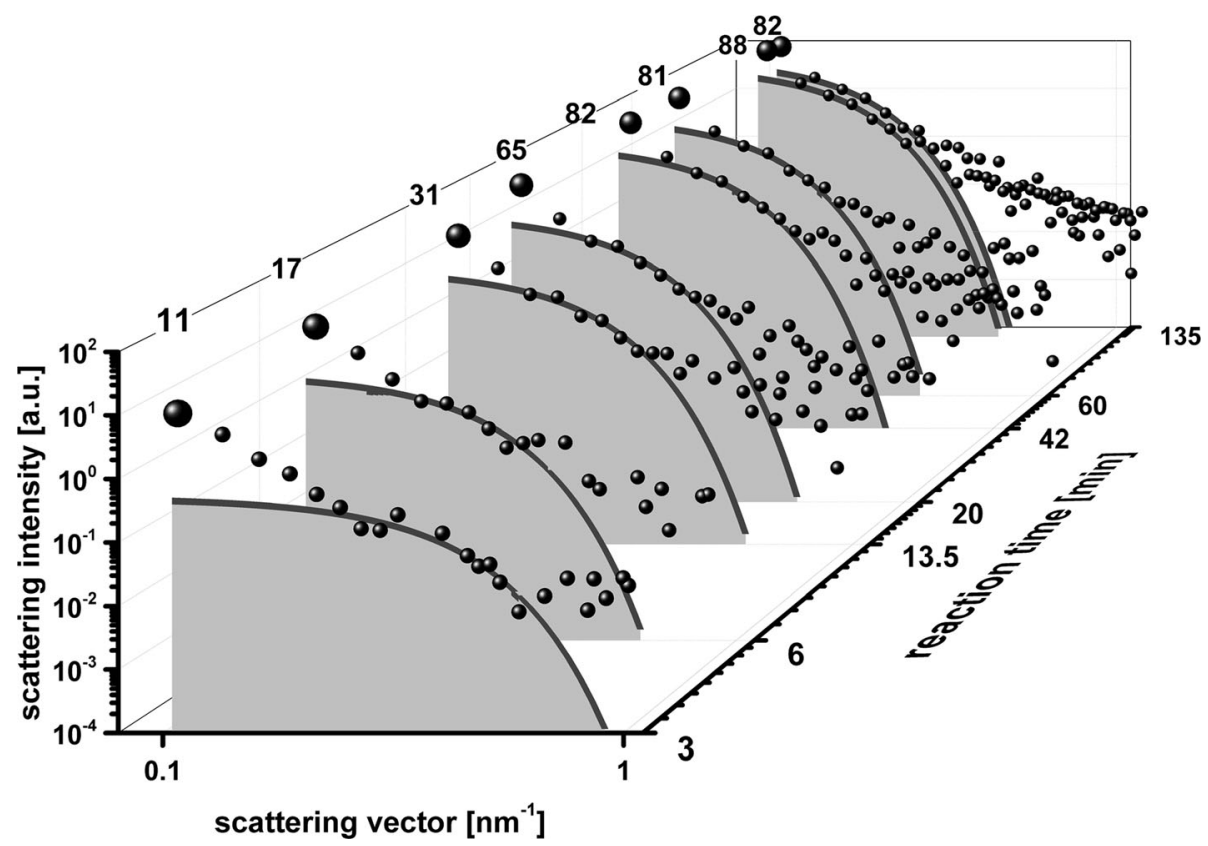

Fig. 6 The scattering intensity of AZO nanocrystals by means of SAXS is plotted over scattering vector $q<1 \mathrm{~nm}^{-1}$ for different reaction times in a $3 \mathrm{D}$ plot. In addition, the respective Guinier plots and the maximum intensities of each scattering curve are marked

crystals were present in an aggregate structure after completion of growth at $t=135 \mathrm{~min}$ (Schnablegger and Singh 2013). Here, $D_{\mathrm{fm}}(t=135 \mathrm{~min})$ was at the upper limit of the value range for determining mass fractional dimensions in Eq. (5), containing information about the relative packing density of the agglomerates investigated. The higher the $D_{\mathrm{fm}}$, the higher the packing density of the agglomerates, from which a maximum packing density could be derived for the AZO crystal system studied in Fig. 8 at $t=$ 135 min (Virtanen et al. 2004). Furthermore, the fractal dimension of the primary particle surface $D_{\mathrm{fS}}(t=135 \mathrm{~min}) \approx 2.1$ was extracted from the slope of the power law for $q>q^{*}$ in Fig. 8, indicating a smooth surface of spherical AZO primary nanocrystals after finishing their growth with respect to the limited range $2<D_{\mathrm{fS}}<3$. To investigate the behavior of fractal properties during growth of primary AZO nanocrystals in detail, the respective fractal dimensions of surface and mass $\left(D_{\mathrm{fs}} ; D_{\mathrm{fm}}\right)$ were determined from the scattering curves in Fig. 6 for each reaction time step in the range $3 \mathrm{~min} \leq t \leq$ $135 \mathrm{~min}$ using the power law as shown before and subsequently plotted versus $t$ in Fig. 9 .

Here, a steady increase of $D_{\mathrm{fm}}$ in the range $2.5<D_{\mathrm{fm}}$ $<3$ (see Eq. (5)) could be observed with increasing reaction progress. Within this range, the primary $\mathrm{AZO}$ crystals were always in an agglomerated state, but its packing density increased linearly with progressive reaction time. However, it could not be completely ruled out weather the necessary sample purification steps for SAXS measurement had an additional influence on the aggregation state of the investigated AZO nanocrystals. Therefore, the measured values were considered only relative to each other in order to demonstrate that a densification process has been occurred during the growth process of primary AZO nanocrystals. Consequently, the time-resolved mass fractal analysis by means of SAXS provided clear evidence to support the growth behavior adopted in the "Growth behavior of AZO using kinetic model and TEM" section, which also has been observed a densification process of originally primary crystals to aggregates with remaining internal grain boundaries from TEM analysis in Fig. 5. In contrast to $D_{\text {fim }}$ in Fig. 9, $D_{\mathrm{fS}}$ showed an exponential decrease with increasing $t$ within the fractal range $2 \leq$ $D_{\mathrm{fS}} \leq 3$ (see Eq. (6)), indicating a smoothing process of the primary crystal surface during crystal growth. With regard to the time interval $40 \mathrm{~min} \leq t \leq 60 \mathrm{~min}$, no further significant change in $D_{\mathrm{fS}}$ could be observed here, indicating the completion of the surface smoothing process within a similar range as the overall synthesis process previously observed from the kinetic model in the "Kinetics of AZO nanocrystal synthesis using QPA" 


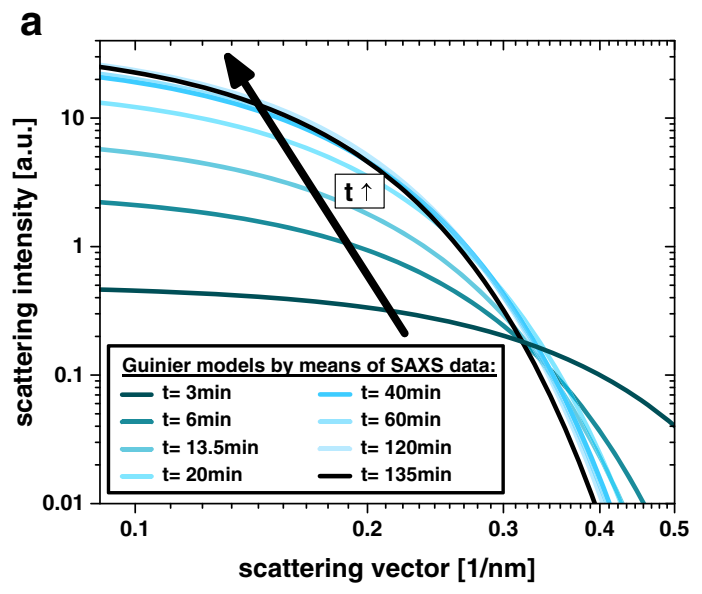

b

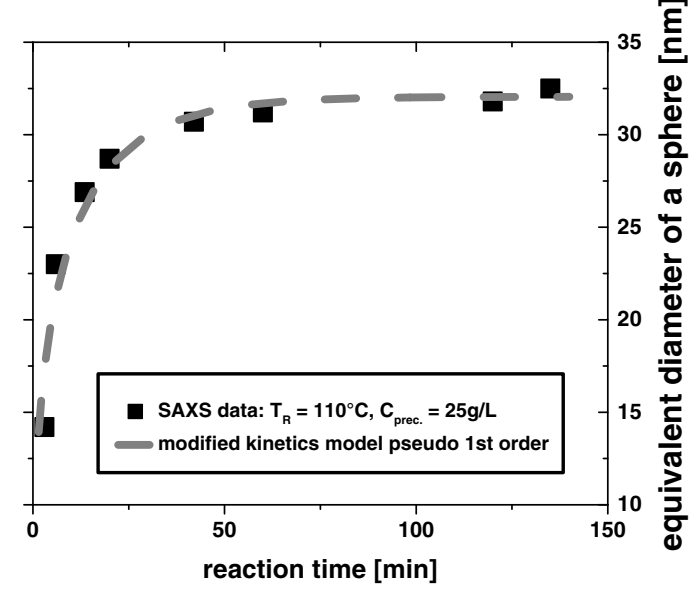

Fig. 7 Guinier models by means of SAXS data from Fig. 6 (a) and the equivalent diameter of a sphere, extracted from Guinier models, is plotted over reaction time and approximated by the modified kinetics model described in the "Kinetic model describing AZO nanocrystal synthesis" section (b)

section. The continued densification process within this time interval, which can be seen from the linear course of $D_{\mathrm{fm}}$, may strongly indicate that processes of rearrangement of primary crystals still occurred even after

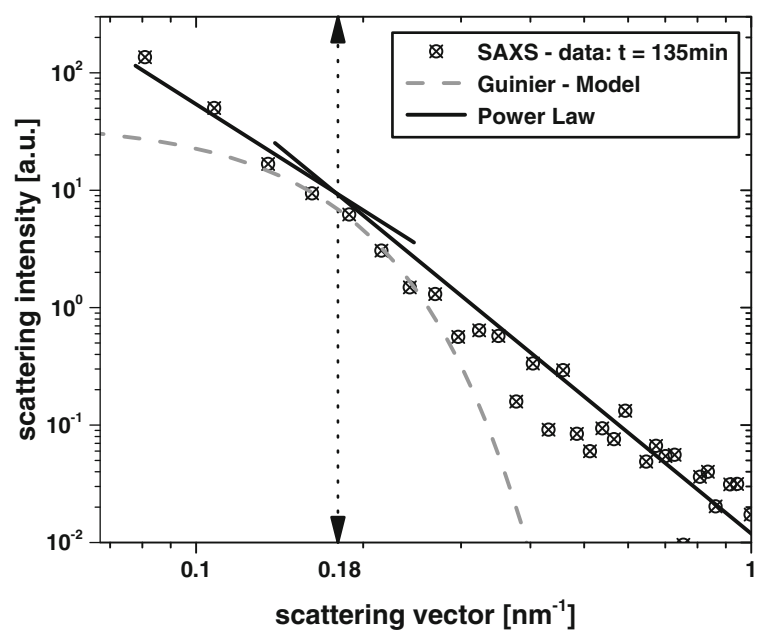

Fig. 8 Scattering intensity of AZO nanocrystals by means of SAXS, sampled after $t=135 \mathrm{~min}$, is plotted over scattering vector in a double logarithmic way (dots), the respective Guinier model (dashed line), and the power law functions for $q<q^{*}$ and $q>q^{*}$, while $q^{*}$ sets the change in slope of the total scattering curve

completion of the primary crystal growth and the surface smoothing processes under the influence of thermal energy and time, as reported in case of titanium dioxide by Dalmaschio and Leite (2012).

Growth model for AZO nanocrystal synthesis via the benzylamine route

Based on all findings gained in this work, a nonclassical crystal growth model describing morphological changes during the synthesis of AZO mesocrystals via the benzylamine route was generally derived at the investigated process conditions $\left(T_{\mathrm{R}}=110^{\circ} \mathrm{C} ; C_{\mathrm{prec}}=\right.$ $25 \mathrm{~g} \mathrm{~L}^{-1}$ ) and illustrated in Fig. 10. In general, the growth behavior of AZO nanocrystals could be divided in three growth stages.

In the first stage of growth in Fig. 10, for $t \leq 3 \mathrm{~min}$ in the context of the examined process conditions, a

Table 3 Overview of the maximum growth ratios of AZO from TEM data $\left(X^{\mathrm{TEM}}\right)$, SAXS data $\left(X^{\mathrm{SAXS}}\right)$, and kinetics model data $\left(X^{\mathrm{Kin} .}\right)$ and including the respective raw data at the earliest stage of growth $(t=3 \mathrm{~min})$ and at the end of growth $(t=135 \mathrm{~min})$

\begin{tabular}{|c|c|c|c|}
\hline Method & $t=3 \mathrm{~min}$ & $t=135 \mathrm{~min}$ & $\begin{array}{l}\text { Max. growth ratio } \\
X^{i}[-]\end{array}$ \\
\hline $\begin{array}{l}\text { TEM data: } \\
d_{\mathrm{S},}, \quad \text { TEM }[\mathrm{nm}]\end{array}$ & $11.7 \pm 25 \%$ & $72 \pm 10 \%$ & 6 \\
\hline $\begin{array}{l}\text { SAXS data: } \\
d_{\mathrm{S}, \text { SAXS }}[\mathrm{nm}]\end{array}$ & $14.2 \pm 17 \%$ & $32.5 \pm 3 \%$ & 2 \\
\hline $\begin{array}{l}\text { Kinetics model data: } \\
C\left[\mathrm{~g} \mathrm{~L}^{-1}\right]\end{array}$ & $1.1 \pm 5 \%$ & $7.3 \pm 1 \%$ & 2 \\
\hline
\end{tabular}


Fig. 9 Fractal mass and surface dimensions $D_{\mathrm{fm}}, D_{\mathrm{fS}}$ of the primary AZO nanocrystals by means of SAXS dependend on reaction time at constant process conditions $\left(T_{\mathrm{R}}=110^{\circ} \mathrm{C} ; C_{\mathrm{E}}=\right.$ $25 \mathrm{~g} \mathrm{~L}^{-1}$ ) and the appropriate fit functions to illustrate its timedependent course

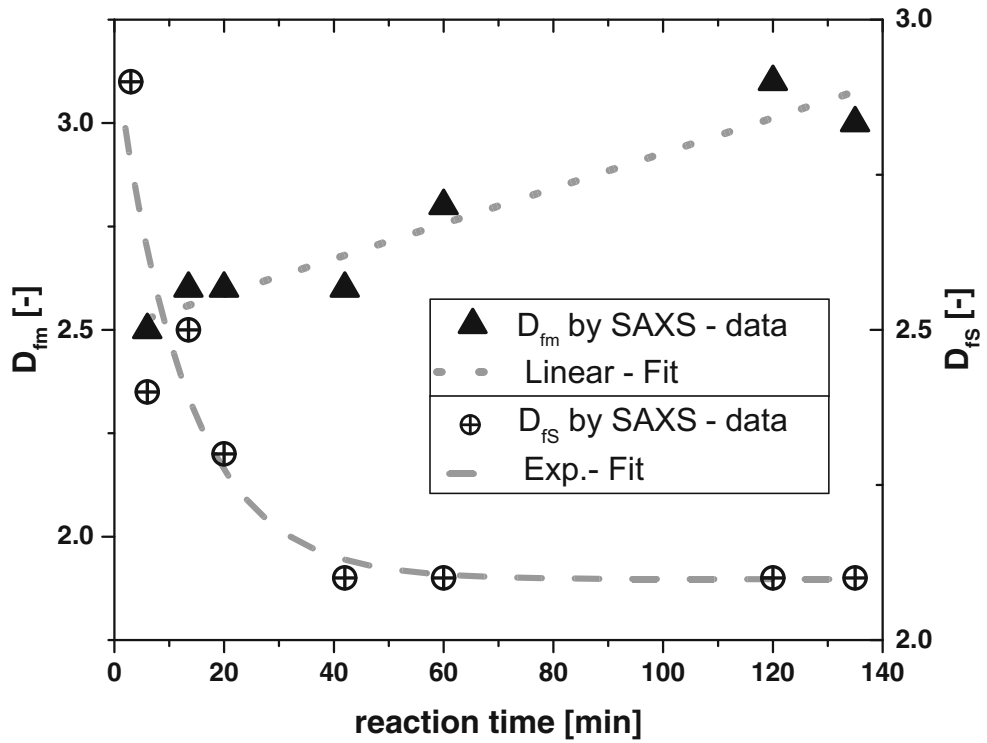

homogeneous nucleation followed by an uniform nuclei growth leads to single AZO primary nanocrystals with roughened surfaces, as confirmed by TEM analysis of Fig. 4a in the "Growth behavior of AZO using kinetic model and TEM" section and the SAXS evaluation of Fig. 6 in the "Morphological analysis of crystal growth using SAXS" and "Fractal analysis during AZO growth using SAXS" sections. In the second stage of Fig. 10, several mechanisms occur simultaneously in the range $3 \mathrm{~min}<t<60 \mathrm{~min}$. It includes an exponential growth of the primary AZO nanocrystals to their final size following pseudo-first-order kinetics until the fully turnover of zinc precursor, which could be obtained from QPA data in the "Kinetics of AZO nanocrystal synthesis using QPA" section and the SAXS data in the "Morphological analysis of crystal growth using SAXS" section. Due to the lack of a stabilizer, an oriented agglomeration of the primary crystals into hexagonal-shaped AZO mesocrystals with internal grain boundaries achieving an energetically favorable arrangement could be observed by TEM and fractal analysis with SAXS in the "Growth behavior of AZO using kinetic model and TEM" and "Fractal analysis during AZO growth using SAXS" sections. In addition, a smoothing process of the primary crystal surface and a densification process of the AZO mesocrystals take place during the synthesis occurs, which could be observed in detail by fractal analysis with SAXS in the "Fractal analysis during AZO growth using SAXS" section. In the third stage, in our case within the range $60 \mathrm{~min}<t<135 \mathrm{~min}$, the densification and orientation process still occur after the overall synthesis process and thus the growth of the AZO primary crystals has already ended, which could be observed by comparison of time-resolved fractal

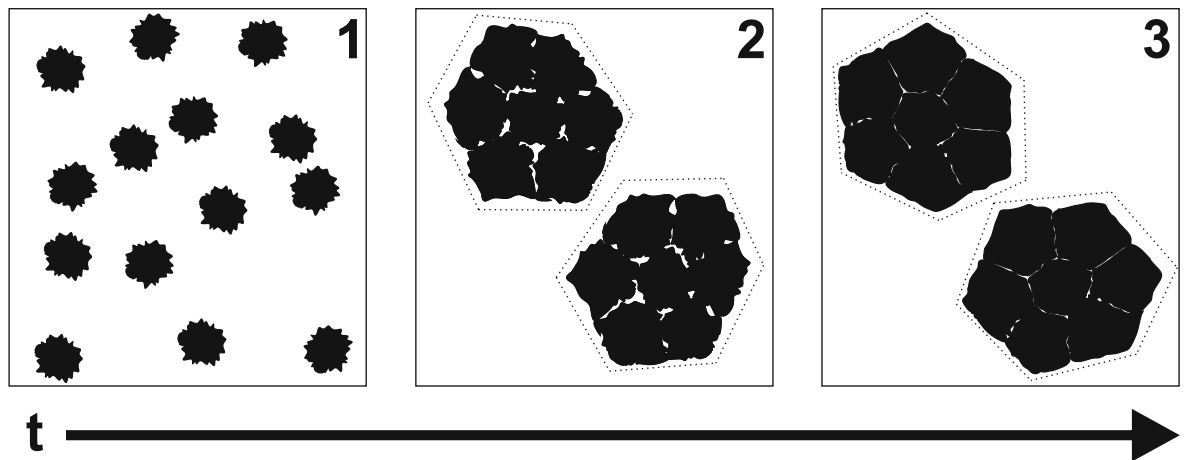

Fig. 10 Growth model for the formation of $A Z O$ nanocrystals during non-aqueous sol-gel synthesis process via the benzylamine route, which is generally divided into three growth stages 
mass analysis from SAXS data in the "Fractal analysis during AZO growth using SAXS" section with kinetics properties of the total synthesis process from QPA data in the "Kinetics of AZO nanocrystal synthesis using QPA" section. After finishing the densification and orientation processes, oriented AZO mesocrystals with a typical hexagonal shape and thus a high similarity to the wurtzite structure of a $\mathrm{ZnO}$ single crystal (Özgür et al. 2005), smooth surfaces and remaining internal grain boundaries arise (see Figs. $4 \mathrm{~b}$ and 5a). Similar findings of densification and orientation processes during the non-aqueous formation of AZO nanolayers also have been observed by the work of Luo et al. (2012).

\section{Conclusions}

The novelty in this work is a developed method of showing time-resolved accessibility of measurement techniques to nanometer scale leading to a detailed growth model, which describes different growth stages in the formation of hexagonal-shaped AZO mesocrystals during the synthesis via the benzylamine route. Here, the obtained growth model includes different particle formation mechanisms, such as primary particle growth, surface smoothing, oriented agglomeration, and densification processes, which were previously obtained by means of SAXS and TEM analyses and finally supported with a pseudo-first-order process kinetics of the total synthesis process based on a new QPA method using a lab-scale SAXS camera. In detail, a newly developed and gravimetrically successfully validated QPA method was first introduced to record the consumption of the zinc precursor in a time-resolved manner, thus deriving a pseudo-first-order process kinetics for the overall synthesis process of AZO nanocrystals via the benzylamine route. By the knowledge of the previously validated process kinetics, on the one hand time intervals with high expected crystal growth rates and on the other hand the termination of crystal growth could be estimated very well, deriving optimal sampling times for the further structural analysis of the AZO growth behavior. In the context of the structural analysis of AZO, the introduction of a maximum growth factor as a comparative factor between TEM analysis and the overall process kinetics at the earliest detectable and a finished growth state successfully demonstrated that not a classical crystallization of pure single crystals but a non-classical crystallization mechanism into hexagonal-shaped AZO mesocrystals with internal grain boundaries occurred. Furthermore, a more detailed and comprehensive insight into the growth behavior of the AZO mesocrystals in terms of primary particle size and fractal structures could be obtained by using a self-built SAXS laboratory camera. Based on SAXS data, the obtained exponential change of primary particle size of AZO mesocrystals during growth could be obtained despite agglomeration, subsequently verified with TEM and successfully validated with the pseudo-first-order kinetics of the total $A Z O$ synthesis process. Moreover, the fractal analysis based on SAXS data proved that two processes occurred simultaneously during AZO crystal growth: a smoothing process of the primary crystal surfaces on the one hand and a densification process of the aggregates to mesocrystals with remaining internal grain boundaries on the other hand. Finally, using previously obtained TEM, SAXS, and kinetics data based on the synthesis conditions studied, a new general growth model for AZO nanocrystal synthesis via the benzylamine route was postulated getting an in-depth insight in particlebased formation mechanisms in the nanoscale leading to fractal and hexagonal-shaped AZO mesocrystals. Looking ahead, this work will serve as fundamental work in nanoparticle research to demonstrate the accessibility of measurement methods to nanoscale area obtaining comprehensive insights in particle formation mechanisms.

Acknowledgements We express our immense thanks to $\mathrm{Mr}$. Tim Köhler and Mr. Florian Kaiser for the experimental assistance and Mrs. Sabrina Zellmer for fruitful discussions.

Funding The research leading to these results has received funding from the German Research Foundation (DFG Ni 414/ 24-1 and Ga 1492/9-1).

\section{Compliance with ethical standards}

Conflict of interest The authors declare that they have no conflict of interest.

\section{References}

Ashcroft NW, Lekner J (1966) Structure and resistivity of liquid metals. Phys Rev 145:83-90

Beaucage G (1995) Approximations leading to a unified exponential/power-law approach to small-angle scattering. J 
Appl Crystallogr 28:717-728. https://doi.org/10.1107 /S0021889895005292

Boukari H, Lin JS, Harris MT (1997) Small-angle X-ray scattering study of the formation of colloidal silica particles from alkoxides: primary particles or not? J Colloid Interface Sci 194: 311-318. https://doi.org/10.1006/jcis.1997.5112

Cölfen H, Antonietti M (2005) Mesocrystals: inorganic superstructures made by highly parallel crystallization and controlled alignment. Angew Chem Int Ed 44:5576-5591

Cushing BL, Kolesnichenko VL, O'Connor CJ (2004) Recent advances in the liquid-phase syntheses of inorganic nanoparticles. Chem Rev 104:3893-3946. https://doi.org/10.1021 /cr030027b

Dalmaschio CJ, Leite ER (2012) Detachment induced by Rayleigh-instability in metal oxide nanorods: insights from TiO2. Cryst Growth Des 12:3668-3674. https://doi. org $/ 10.1021 / \operatorname{cg} 300473 \mathrm{u}$

Ellinger CR, Nelson SF (2014) Selective area spatial atomic layer deposition of $\mathrm{ZnO}, \mathrm{Al} 2 \mathrm{O} 3$, and aluminum-doped $\mathrm{ZnO}$ using poly (vinyl pyrrolidone). Chem Mater 26:1514-1522

Garnweitner G, Grote C (2009) In situ investigation of molecular kinetics and particle formation of water-dispersible titania nanocrystals. Phys Chem Chem Phys 11:3767-3774

Garnweitner G, Tsedev N, Dierke H, Niederberger M (2008) Benzylamines as versatile agents for the one-pot synthesis and highly ordered stacking of anatase nanoplatelets. Eur J Inorg Chem 2008:890-895

Glatter O, Kratky O (eds) (1982) Small-angle X-ray scattering. Academic Press, New York

Goertz V, Gutsche A, Dingenouts N, Nirschl H (2012) Small-angle $\mathrm{X}$-ray scattering study of the formation of colloidal $\mathrm{SiO} 2$ stober multiplets. J Phys Chem C 116:26938-26946

Guo X, Gutsche A, Nirschl H (2013a) SWAXS investigations on diffuse boundary nanostructures of metallic nanoparticles synthesized by electrical discharges. J Nanopart Res 15:2058

Guo X, Gutsche A, Wagner M, Seipenbusch M, Nirschl H (2013b) Simultaneous SWAXS study of metallic and oxide nanostructured particles. J Nanopart Res 15:1559. https://doi. org/10.1007/s11051-013-1559-8

Gutsche A, Daikeler A, Guo X, Dingenouts N, Nirschl H (2014) Time-resolved SAXS characterization of the shell growth of silica-coated magnetite nanocomposites. J Nanopart Res 16: 2475. https://doi.org/10.1007/s11051-014-2475-2

Gutsche A, Meier M, Guo X, Ungerer J, Nirschl H (2017) Modification of a SAXS camera to study structures on multiple scales. J Nanopart Res 19:321

Ismail B, Abaab M, Rezig B (2001) Structural and electrical properties of $\mathrm{ZnO}$ films prepared by screen printing technique. Thin Solid Films 383:92-94

Jia B, Gao L (2008) Growth of well-defined cubic hematite single crystals: oriented aggregation and Ostwald ripening. Cryst Growth Des 8:1372-1376. https://doi.org/10.1021 $/ \operatorname{cg} 070300 t$

Jiang X, Wong FL, Fung MK, Lee ST (2003) Aluminum-doped zinc oxide films as transparent conductive electrode for organic light-emitting devices. Appl Phys Lett 83:1875-1877. https://doi.org/10.1063/1.1605805

Karlak R, Burnett D (1966) Quantitative phase analysis by X-ray diffraction. Anal Chem 38:1741-1745

Kelchtermans A, Elen K, Schellens K, Conings B, Damm H, Boyen HG, D'Haen J, Adriaensens P, Hardy A, van Bael
MK (2013) Relation between synthesis conditions, dopant position and charge carriers in aluminium-doped $\mathrm{ZnO}$ nanoparticles. RSC Adv 3:15254-15262

Livage J, Henry M, Sanchez C (1988) Sol-gel chemistry of transition metal oxides. Prog Solid State Chem 18:259-341

Ludi B, Süess MJ, Werner IA, Niederberger M (2012) Mechanistic aspects of molecular formation and crystallization of zinc oxide nanoparticles in benzyl alcohol. Nanoscale 4:1982 1995

Luo L, Rossell MD, Xie D, Erni R, Niederberger M (2012) Microwave-assisted nonaqueous sol-gel synthesis: from Al: $\mathrm{ZnO}$ nanoparticles to transparent conducting films. ACS Sustain Chem Eng 1:152-160

Minami T (2005) Transparent conducting oxide semiconductors for transparent electrodes. Semicond Sci Technol 20:S35S44

Nie D, Xue T, Zhang Y, Li X (2008) Synthesis and structure analysis of aluminum doped zinc oxide powders. Sci China Ser B Chem 51:823-828

Niederberger M (2007) Nonaqueous sol-gel routes to metal oxide nanoparticles. Acc Chem Res 40:793-800

Niederberger M, Cölfen H (2006) Oriented attachment and mesocrystals: non-classical crystallization mechanisms based on nanoparticle assembly. Phys Chem Chem Phys 8:32713287

Olliges-Stadler I, Rossell MD, Süess MJ, Ludi B, Bunk O, Pedersen JS, Birkedal H, Niederberger M (2013) A comprehensive study of the crystallization mechanism involved in the nonaqueous formation of tungstite. Nanoscale 5: $8517-8525$

Özgür Ü et al (2005) A comprehensive review of $\mathrm{ZnO}$ materials and devices. J Appl Phys 98:11

Patil A, Dighavkar C, Borse R (2011) Al doped ZnO thick films as CO2 gas sensors. J Optoelectron Adv Mater 13:1331-1337

Penn RL, Soltis JA (2014) Characterizing crystal growth by oriented aggregation. CrystEngComm 16:1409-1418

Pinna N, Niederberger M (2008) Surfactant-free nonaqueous synthesis of metal oxide nanostructures. Angew Chem Int Ed 47: 5292-5304

Pinna N, Garnweitner G, Antonietti M, Niederberger M (2005) A general nonaqueous route to binary metal oxide nanocrystals involving a $\mathrm{C}-\mathrm{C}$ bond cleavage. J Am Chem Soc 127:5608-5612

Porod G (1951) Die Röntgenkleinwinkelstreuung von dichtgepackten kolloiden Systemen. Kolloid-Zeitschrift 124:83-114

Schmidt P (1991) Small-angle scattering studies of disordered, porous and fractal systems. J Appl Crystallogr 24:414 435 . https://doi.org/10.1107/S0021889891003400

Schnablegger H, Singh Y (2013) The SAXS guide. Anton Paar $\mathrm{GmbH}$

Singh MA, Ghosh SS, Shannon RF Jr (1993) A direct method of beam-height correction in small-angle X-ray scattering. J Appl Crystallogr 26:787-794. https://doi.org/10.1107 /S0021889893005527

Song RQ, Cölfen H (2010) Mesocrystals-ordered nanoparticle superstructures. Adv Mater 22:1301-1330

Soofivand F, Tavakoli F, Salavati-Niasari M (2014) Synthesis and characterization of $\mathrm{Zn}(\mathrm{acac}) 2$ one-dimensional nanostructures by novel method. Sumy State University 
Strachowski T, Grzanka E, Lojkowski W, Presz A, Godlewski M, Yatsunenko S, Matysiak H, Piticescu RR, Monty CJ (2007) Morphology and luminescence properties of zinc oxide nanopowders doped with aluminum ions obtained by hydrothermal and vapor condensation methods. J Appl Phys 102: 073513

Stubhan T, Oh H, Pinna L, Krantz J, Litzov I, Brabec CJ (2011) Inverted organic solar cells using a solution processed aluminum-doped zinc oxide buffer layer. Org Electron 12: 1539-1543

Virtanen A, Ristimäki J, Keskinen J (2004) Method for measuring effective density and fractal dimension of aerosol agglomerates. Aerosol Sci Technol 38:437-446

Wang ZL (2004) Nanostructures of zinc oxide. Mater Today 7:2633. https://doi.org/10.1016/S1369-7021(04)00286-X
Yoon M, Lee S, Park H, Kim H, Jang M (2002) Solid solubility limits of Ga and Al in ZnO. J Mater Sci Lett 21:1703-1704

Zellmer S, Kockmann A, Dosch I, Temel B, Garnweitner G (2015) Aluminum zinc oxide nanostructures with customized size and shape by non-aqueous synthesis. CrystEngComm 17: 6878-6883. https://doi.org/10.1039/c5ce00629e

Zhang Q, Liu S-J, Yu S-H (2009) Recent advances in oriented attachment growth and synthesis of functional materials: concept, evidence, mechanism, and future. J Mater Chem 19:191-207

Publisher's note Springer Nature remains neutral with regard to jurisdictional claims in published maps and institutional affiliations. 